\title{
The ascendance of microphysiological systems to solve the drug testing dilemma
}

The development of drugs is a process obstructed with manifold security and efficacy concerns. Although animal models are still widely used to meet the diligence required, they are regarded as outdated tools with limited predictability. Novel microphysiological systems intend to create systemic models of human biology. Their ability to host 3D organoid constructs in a controlled microenvironment with mechanical and electrophysiological stimuli enables them to create and maintain homeostasis. These platforms are, thus, envisioned to be superior tools for testing and developing substances such as drugs, cosmetics and chemicals. We will present reasons why microphysiological systems are required for the emerging demands, highlight current technological and regulatory obstacles, and depict possible solutions from state-of-the-art platforms from major contributors.

Lay abstract: Microphysiological systems are devices constructed for the cocultivation of miniaturized human organ equivalents. They are commonly placed into a continuous stream of nutrient solution. The microphysiological tools aim to reshape current development, toxicity testing and efficacy assessment of therapeutic agents, food additives, chemicals and environmental pollutants. We are on the verge of initiating a paradigm shift away from established, but often misleading animal and single tissue culture techniques toward the generation of predictive data for a compound's safety and efficacy prior to its exposure to humans.

First draft submitted: 6 January 2017; Accepted for publication: 14 February 2017; Published online: 31 March 2017

Keywords: body-on-a-chip • drug testing • human-on-a-chip • microphysiological system - multi-organ-chip

\section{The drug testing dilemma}

Drug developers are facing an ever-increasing problem - their costs for releasing a new product are skyrocketing. The authorization process including clinical and preclinical trials is not only complicated and highly restrained, but takes an exceptionally long time. It is estimated that the development, testing and authorization of a single drug candidate currently takes up to 13.5 years [1] and costs an average of US $\$ 2.5$ billion [2]. The cost explosion over the past decade has naturally several reasons. One major cost driver is the fact that of 100 substances entering clinical testing in humans, only ten will be allowed onto the market $[2,3]$. The high attrition rate is cross-financed by those few substances. Approximately half of the candidates excluded are retracted due to safety concerns [4,5]. Apart from deprecating an investment, there is an eminent risk for any proband in the clinical trials, as was yet again obvious regarding a fatality in a Phase I clinical trial in early 2016 [6,7]. The regulatory safety requirements are indubitably a main driver for the increase in costs. However,
Eva-Maria Dehne*,1, Tobias Hasenberg ${ }^{1} \&$ Uwe Marx ${ }^{1}$ 'TissUse GmbH, Oudenarder Straße 16, 13347 Berlin, Germany *Author for correspondence: Tel.: +4930513026450 Fax: +49 30513026401 eva.dehne@tissuse.com 
nobody is inclined to sacrifice risk assessment for commercial optimization.

Consequently, the substance testing should be optimized in such a way that candidate drugs are retracted from the risk assessment as early as possible [1]. Therefore, the drug discovery and the preclinical risk assessment are thought to be the prominent levers for reducing costs (Figure 1).

The difficulties for drug developers and toxicologists are now manifold. First, their test systems are obviously not optimal to predict safety and efficacy in humans properly. Simultaneously and second, safety requirements will rather intensify than halt. Third, the amount of substances awaiting testing will increase [8]. Most importantly, their complexity will be boosted as more and more biopharmaceuticals are in development $[9,10]$, along with mixtures that have effects unpredictable from the respective single substance knowledge [11].

Substances are tested currently, first and foremost, either in animal models - mostly rodents - or with human cell cultivation techniques. Toxicologists might refer to them as proxy and reductionist models, respectively [12]. The former permits access to an entire organism with all its immunity, microenvironments, organ-organ interactions and behavioral responses to any given substance. Some might regard them as the gold standard, but they have more than once proven to be inaccurate due to their phylogenetic distance and methodological flaws [13-17]. In fact, widely used drugs, such as aspirin or paracetamol, would not be approved with current animal models [18]. Their predictability is questionable as they are obviously not human. In the words of Marcel Leist and Thomas Hartung: "Humans are no $70-\mathrm{kg}$ mice" [16]. Apart from the candidate drugs that were incorrectly allowed for human testing, there may be substances equally incorrectly repealed waiting to be retrieved like a forgotten treasure.

In vitro cultivation - the reductionist model - is usually based on human cells. It is a field of constant innovations and new technologies, especially in the area of tissue engineering. The declared ambition is the emulation of the functionality of any organ. These tissue models are often dedicated for regenerative therapies. They are equally desired in substance testing. Nevertheless, focusing on a specific tissue will not overcome the dilemma described, no matter how close-to-physiological a model becomes. Toxicity often emanates from complex interactions across tissues. The diversity of metabolites is especially regarded as critical. The drug Terfenadine, for example, is only activated in the liver [19]. However, the activated form simultaneously becomes cardiotoxic. Regarding the fatality case mentioned earlier, metabolites of the drug candidate remain a probable cause for the dire outcome of the clinical trial [7].

Moreover, as the cell culture is often a reduced, outof-context model, it is hard to relate concentrations of substances back to the human case. The exposition in vivo is often either unknown or hard to predict [12]. Similarly, the influence of absorption, distribution, metabolism and excretion (ADME) needs to be regarded. The extrapolation of the data gathered to the in vivo situation is, therefore, again highly questionable. Hence, conclusions gained from cell culture models usually give rise to yes or no answers rather than dose information.

The dilemma for toxicologist is now the following: while regulations, quantity and complexity of the substances increase continuously, current test systems are partly outdated, partly weak in predictability. The estimation of safe starting and maximum doses for clinical phases in humans are founded on mere leap-of-faith decisions than on well transferable data.

In fact, the issues concerning substance testing are encountered not only by the pharmaceutical industry. In 2013, the European Union banned all animal-based experimentation for newly approved cosmetic products. Additionally, new cosmetics that have been developed and tested in animal-based experiments were excluded from the European market [20]. Although it does not prohibit animal tests, the REACH regulation (Registration, Evaluation, Authorization and Restriction of Chemicals) is also thought to have contributed to the development of alternative tests. It demands transparency of toxicological datasets, encourages alternative methods and tries to prohibit the reassessment of toxic queries in animals by demanding approval by the agency $[8,21]$.

The rise of microphysiological systems (MPS), about a decade ago, marks a turning point. They allowed the combination of highly sophisticated human organ models and mimic their systemic interplay. The variety of solutions and developments underscore the importance and potential ascribed to these devices - not only by scientists, but also by the industrial end users. Other economic and scientific fields, such as food or ecology, will profit similarly from human MPS. If these devices convince industry and regulators alike, they will initiate a paradigm shift away from obsolete in vivo experiments toward new approaches of substance development and risk assessment.

\section{Microphysiological systems in the context of cell culture technology}

In short, MPS are microfluidic devices designed to support a physiological environment for in vitro cultures and, finally, emulate biology. An MPS in its sim- 


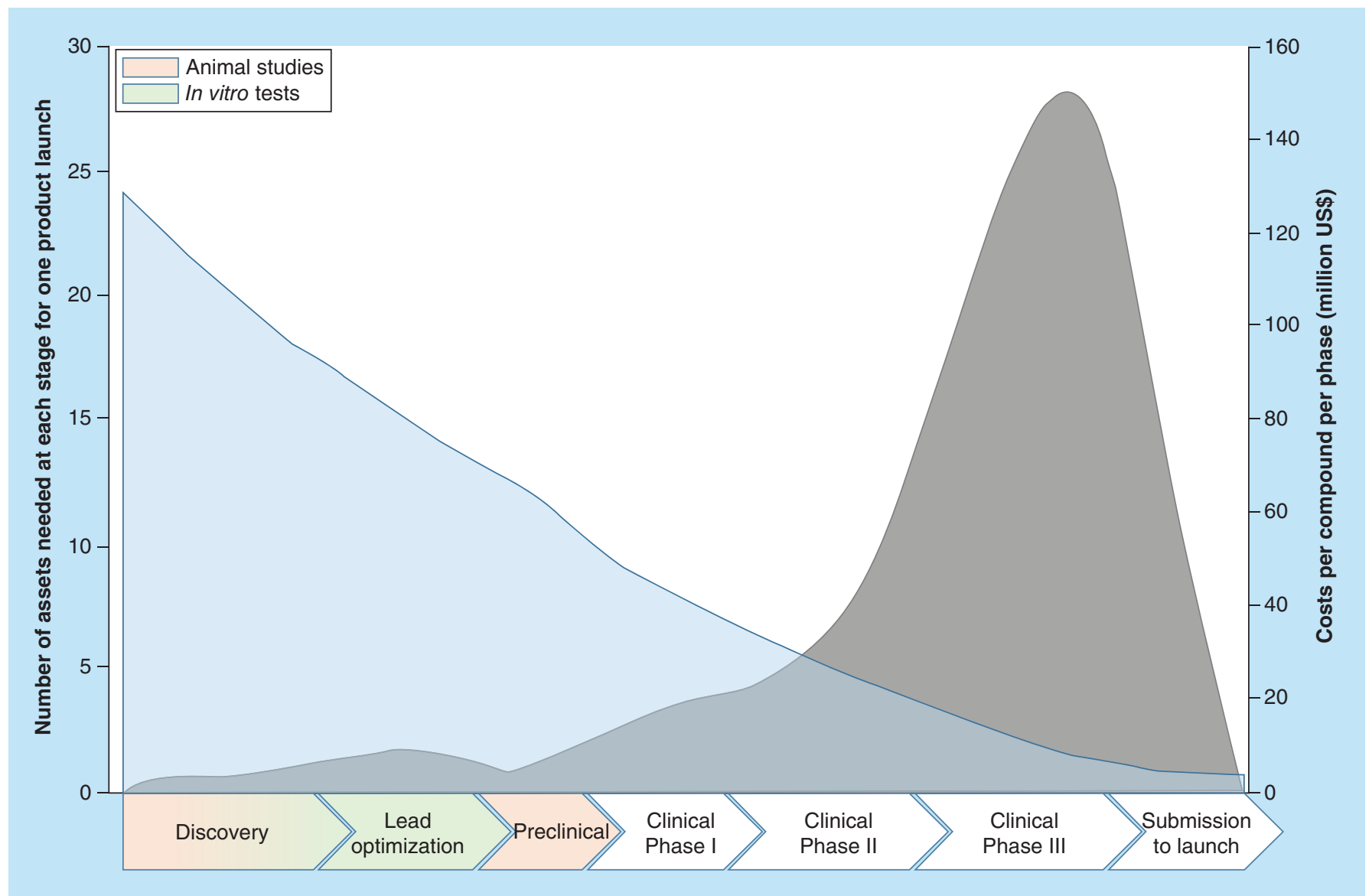

Figure 1. The drug testing dilemma. The graph illustrates schematically the route from substance development through preclinical and clinical evaluation of toxicity and efficacy until release. The process is estimated to take an average of 13.5 years. Depending on the source, only $4-10 \%$ of the initial drug candidates will become market-ready and will cross-finance the withdrawn substances. Retraction at a late stage becomes especially costly, which is why the developing companies try to improve the predictability in the preclinical phases and resolve inapt drugs (numbers are extracted from Paul et al. [1]).

plest form resembles, for example, the device published by Heemskerk et al. [22]. Here, a perfused channel of $300 \times 52 \mu \mathrm{m}$ lined with endothelial cells emulates a blood vessel's intima. The introduction of a stenosis into the channel simulates plaque formation during atherosclerosis. The increased activity of von Willebrand factor in the stenotic region demonstrates the ability of the MPS to act as a disease model. More complex MPS comprise a network of interconnected microchannels in combination with cocultures of several different organ models [23]. A selection of MPS devices will be presented later.

The MPS are the logical successor of lab-on-a-chip devices ascending at the beginning of the last decade. Different to what the term suggests, these tools do not necessarily comprise microelectronics. Their manufacturing, however, makes frequent use of semiconductor techniques enabling the miniaturization of huge and expensive devices. The MPS do not necessarily profit from the same ease-of-use and point-of-care functionalities. The benefits for MPS lie somewhere else.

The MPS devices, for instance, enable the building up of physiological-like microenvironments for respective cell cultures. In terms of 3D cultivation techniques, the MPS can be designed for hosting and improving such cultures. The perfusion of the systems enacts a proper shear stress environment at physiological intracapillary or interstitial rates. It clears secreted products, permits the interaction of distant cells and the creation of microenvironmental biomolecular gradients. The generation of relevant mechanical cues is a major aspect of these systems, distinguishing them from conventional static in vitro cell cultures; for example, the stretching of lung epithelial cells [24] or the electrophysiological stimulation in cardiac models [25]. The drive to catch up with the physiological template will introduce disease and regenerative models [26]. Such endeavors raise the need for an immune system in MPS, even if partial. The systems enable the incorporation of extracellular matrix components 
or specialized materials, such as ceramics, textiles or polymers, to model stiffness, surface patterns or the microarchitecture.

Furthermore, the miniaturization reduces the amount of cell material and increases the throughput. This is the buzz word for every toxicologist facing an increasing mass of substances in their pipeline. Of course, special care should be taken, as the smallest possible size is not necessarily the most practicable. The MPS engineers are generally interested in subtissue structures - particularly organoids [27].

Organoids are defined either as the smallest functional in vivo units of any organ or as the respective functional in vitro 3D cell accumulations. The former are identical, functionally self-reliant units realizing the most relevant tasks of the entire organ. They typically comprise a large number of cells, different cell types and a defined microstructure. A prime example of an organoid is the liver lobule. In humans, each lobule contains about 1 million cells, about 20 cell types and a complex vessel architecture. One million lobules constitute the liver. Similar sections can be found in every organ [28]. Tissue engineers intend to reconstruct these organoids in MPS.

The maintenance of the cell cultures within the MPS is further improved by functionalities controlling the temperature, $\mathrm{pH}$ and even oxygen [29]. The monitoring capabilities of MPS also allow accurate and continuous observation of the cells. Electrical coupling enables the readout of action potentials in neuronal or cardiac cultures. Transparent bottom plates do not only permit visual insights, but also the optical observation of, for example, oxygen partial pressure [30]. Integrating various sensors and actuators enhances the degree of control substantially.

\section{Challenges \& chances in MPS development}

As described above, the overall goal of all MPS is the emulation of species-specific biology. However, the scales, basic working principles and biological complexities are varied. These are designed to fit different purposes, matching the technical solutions available for the design of the tissue culture compartments and the microfluidics.

The MPS can be generally classified into two factions regarding the generation of fluid flow: passive gravity-driven flow and active pumping. The former was developed regarding standardization, automation and high-throughput testing without the need for extensive external accessories and vast medium reservoirs. These devices are often in the form of a microtiter cell culture plate and its cavities are usually in the same locations as in 96- or 384-plates. Depending on the system, several cavities are microfluidically interconnected and the flow is hydrostatically driven (pumpless perfusion).

Platforms comprising active pumping, on the other hand, generally follow a proprietary design concept. Their less standardized format allows more variability in the dimensions of the organoid compartments, complexity of the microfluidics and integration of sensors. Many devices depend on external roller or syringe pumps, others contain on-chip micropumps [24,31-33]. The former benefit from ease of production and operation, but apart from that, include substantial drawbacks. First, the fluid-to-tissue ratios are beyond the normal physiological range due to the artificially high media volumes required for external pumps. Organ-organ crosstalk is, thus, compromised because the soluble factors are strongly diluted. The use of external tubes, adapters and, hence, many different materials also aggravate issues concerning unspecific substance adsorption. On-chip pumps, on the other hand, are difficult to characterize, especially often due to their pulsatile fluid flow generation.

An extensive review of a recent workshop with members of academia, industry and regulators comprehensively summarizes the concepts and examples for various MPS [34]. In the following, the main challenges and opportunities of both MPS types will be discussed based on commercially available or well-reviewed systems.

\section{The advantages of continuous perfusion}

A long-term culture is often impeded due to loss of function, especially when working with demanding primary cells such as hepatocytes. The nonhomeostatic conditions and sudden media changes in static in vitro cell cultures often lead to a dedifferentiation of cells. Therefore, single-organ engineers aimed early on for a continuous supply of nutrients and removal of 'waste' products that cannot be metabolized by the respective cells and would accumulate potentially harming the cells. Cell culture formats with passive gravity-driven microfluidic flow emerged. The CellASIC Corporation (now part of Merck KGaA, Darmstadt, Germany) was one of the first companies commercializing microtiter plates for continuously perfused monolayer cell cultures. Their system is based on a disposable 96-well plate containing four microfluidic circuits, all sample solutions, media and cells. A network of $4 \times 4 \mu \mathrm{m}$ channels surrounds the entire culture compartment. It maintains the cultured cells in a specific imaging region with exposure to continuous media perfusion [35]. The technology was used previously in a custom-made fluidic layout to cultivate hepatocytes under high-density for over 1 week [36]. The continuous medium flow did not only keep cells viable, but also enhanced the spe- 
cific production of albumin by threefold compared with static cultures. The physiologically relevant nutrient supply and flow dynamics provided the necessary cues for maintaining the differentiated phenotype and function of cells. This study further demonstrated the importance of high cell densities.

Cell-cell communication is essential for cells to sense and respond to their environment. Drug toxicity often disrupts intercellular connections, initiating a cascade of cellular events that leads to loss of function and apoptosis. Therefore, recapitulating cell-cell interactions analogous to their in vivo counterparts is critical for physiologically relevant organ models. However, the cell density under standard in vitro conditions is usually 100 - to 1000 -fold lower than in tissues. Moreover, the commercially available CellASIC ${ }^{\circledR}$ plates are not intended for high cell density culture.

\section{Recognizing the relevance of 3D cocultures \& physical stimulation}

The importance of recapitulating not only the in vivolike spatial arrangement, but also interactions of different cell types was further demonstrated by Linda Griffith and her team at the Massachusetts Institute of Technology (MA, USA) (Figure 2A) [32,37,89]. They generated a hepatic capillary bed, using hepatocytes and nonparenchymal cells (including endothelial cells). By respecting the oxygen supply and demand of the cells as well as the shear stress they emulated tissue-like features. Continuous fluid flow was generated by an on-chip pump. An array of $200-\mu \mathrm{m}$ deep and $300 \times 300-\mu \mathrm{m}$ wide through-holes was seeded with cells. Their perfusion led to the morphogenesis of hepatic sinusoidal structures. Cells with features of fenestrated endothelium could be detected even after a cultivation period of 2 weeks. To the best of our knowledge, it was the first time that delicate structures such as fenestrated endothelium could be retained under in vitro conditions for such a period. The liver chip models many aspects of the liver at a very high level and can support a full viral lifecycle hepatitis B. It is commercialized by $\mathrm{CN}$ Bio Innovations Ltd. (Hertfordshire, UK).

Epithelial barriers are another field of broad interest for toxicologists [38]. It is most important for the safety evaluations in the cosmetics industries. Generally, epithelial models provide evidence for the harmfulness of the applied substances. Furthermore, transport across barriers is regarded, which could lead to unintended local or systemic bioavailability. Absorption studies relate external exposure to internal threshold values in the context of regulatory risk assessment. The pharmaceutical industry, on the other hand, is interested in substance efficacy. Therefore, bioavailability becomes a major concern as well as first-pass metabolism in the skin. Various in vitro epithelial barrier models have already found applications in both research and industry as attractive alternatives to animal testing [38]. However, major challenges regarding the generation of physiologically relevant structural, mechanical, absorptive and transport properties have not yet been solved fully. The application of continuous physical stimuli on such cultures was proposed as beneficial in terms of model maturation.

Donald Ingber and his group at the Wyss Institute for Biologically Inspired Engineering at Harvard University (Boston, USA) presented a microphysiological single-organ system that reconstituted a functional alveolar-capillary interface in 2010 (Figure 2B) [24]. The device exerted cyclic mechanical strain on human alveolar epithelial cells and pulmonary microvascular endothelial cells grown on both sides of a thin, porous and highly flexible polydimethylsiloxane (PDMS) membrane. The strain resulted from the stretching of the membrane and the shear stress from flowing media. The perfusion was driven by an external pump. The device could replicate organ-level responses to physiological inflammatory stimuli, such as bacteria or cytokines. Hence, endothelial cells were activated, which led to the induction of adhesion and transmigration of primary human neutrophils flowing in the capillary channel. It effectively allows the creation of disease models and the investigation of inflammatory dynamics from a completely new perspective [39]. The microfluidic device and its derivatives have since also been applied to mimic other organs and parts of organs, such as the kidney's proximal tubule [40], the vascular endothelium [41,42], the bone-marrow [43] and the gut [44]. For the latter, cycling strain mimicking physiological peristaltic motions was exerted on a layer of Caco-2 cells. The group could show that a columnar epithelium developed and polarized rapidly. The barrier, thus, developed a high integrity toward small molecules. Mimicking the intestine was improved compared with models grown under static conditions. The device is commercially available from Emulate Inc. (MA, USA).

\section{Enabling multitissue interactions}

Combining high cell density culture, continuous perfusion and multitissue coculture was presented by Jos Joore and Paul Vulto, managing directors of the company Mimetas (Leiden, The Netherlands) (Figure 2C) [45]. The company recently developed microfluidic 3D culture systems based on 384-well plates. The proprietary PhaseGuide ${ }^{\mathrm{TM}}$ technology allows a membrane-free, yet controlled, deposition of ECM gels. They can be arranged in orderly layers along a microchannel for medium perfusion. Load- 


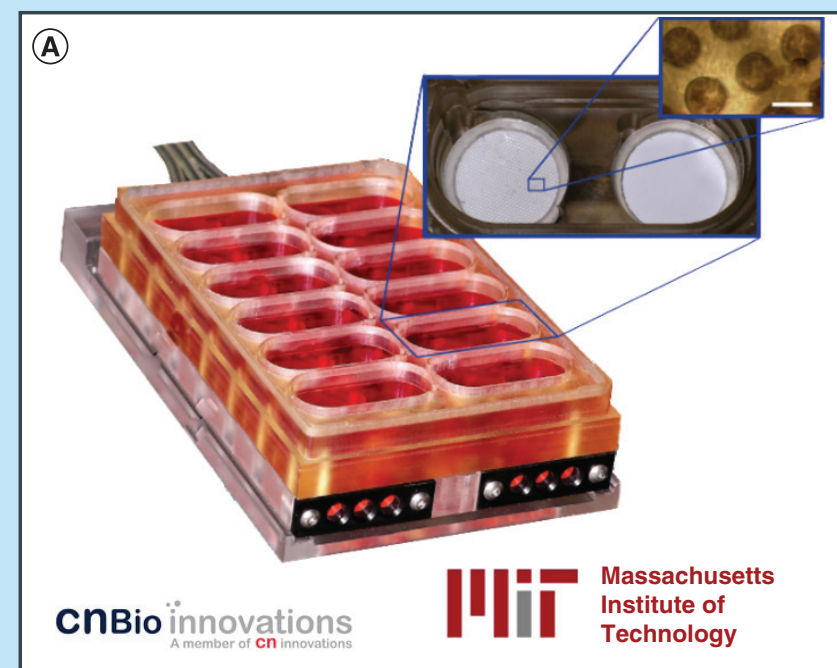

(B)
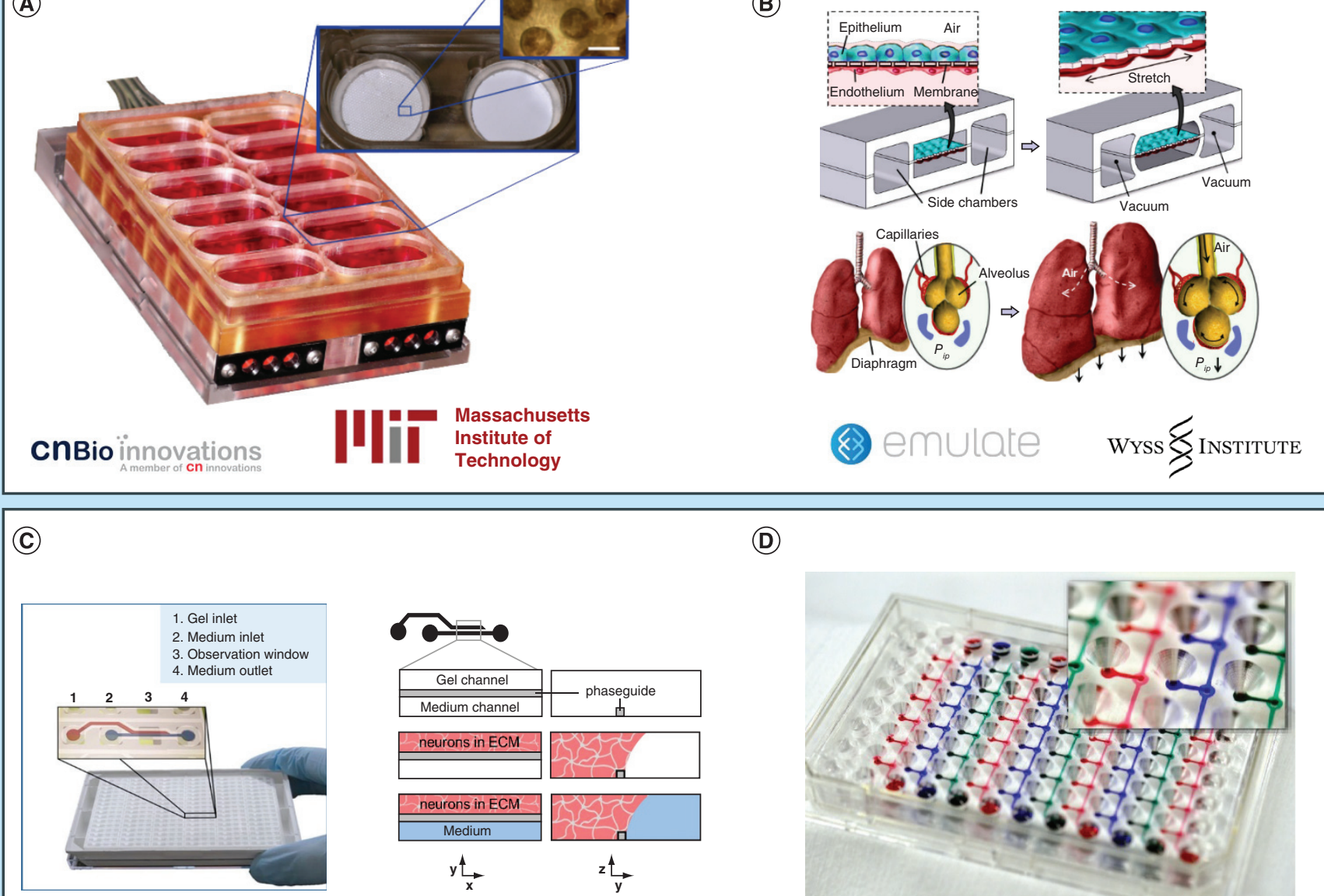

MIMET月E

the organ-on-a-chip company

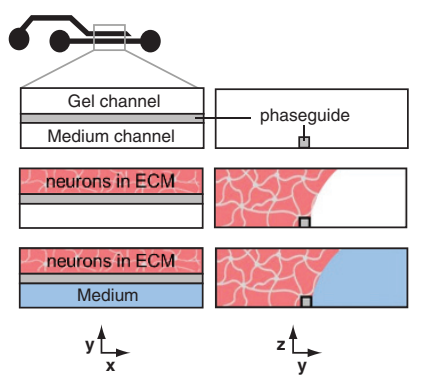

(D)

(E)

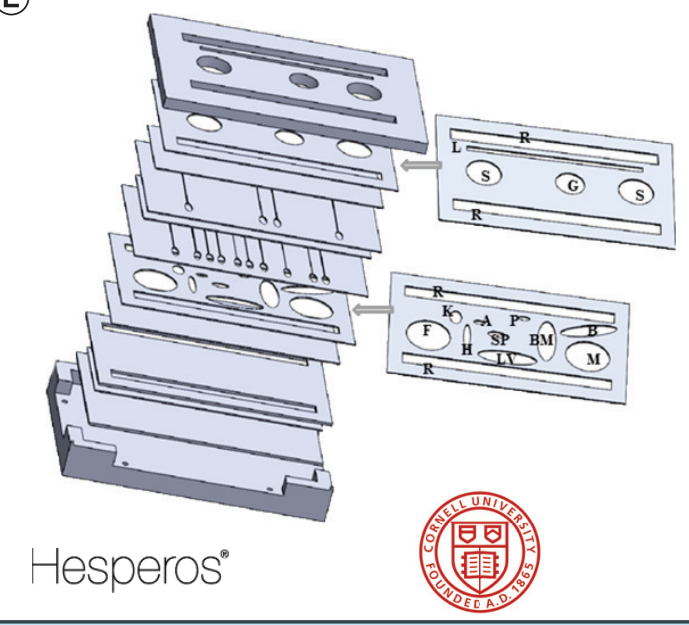

(F)

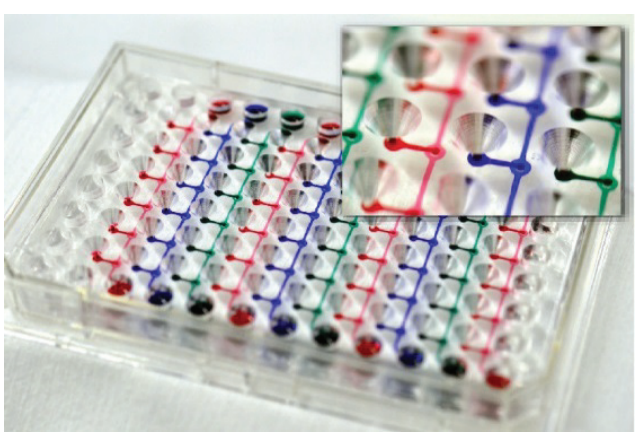

Figure 2. Selection of current microphysiological systems devices with ongoing marketing or commercialization ambitions. The first row shows sophisticated single-organ-specific chip designs. The second row depicts pumpless plate systems with a broad range of applications through a generic layout. The bottom row illustrates multi-organ-chips with individualized cultivation compartments. 
Figure 2. Selection of current microphysiological systems devices with ongoing marketing or commercialization ambitions

(cont. from facing page). (A) The lung-on-a-chip hosts a barrier model containing lung epithelial and endothelial cells (reprinted with permission from Huh et al. [24]). (B) The LiverChip ${ }^{\circledR}$ incorporates primary human liver cells showing extended vitality and in vivo-like morphogenesis over prolonged culture periods (reprinted with permission from Domansky et al. [89]). (C) The OrganoPlates ${ }^{\circledR}$ allow the controlled deposition and subsequent perfusion of hydrogels (reprinted with permission from Wevers et al. [46]). (D) The microfluidic platform accommodates up to eight cell aggregates in compartments that allow communication through the medium (courtesy of Oliver Frey). (E) The body-on-a-chip device designed based on a PBPK model adapts compartment size and microfluidics to the human template considering technological constraints (reprinted with permission from Miller and Shuler [56]). (F) The Four-Organ-Chip was manufactured to mimic adsorption, distribution, metabolism and excretion - information mandatory for the toxic evaluation of any given substance.

PBPK: Physiologically based pharmacokinetic.

ing the gels with cells results in 3D tissue models with physiologically relevant cell densities. As the gels are in direct contact with each other or the media channel, interactions without physical barriers are possible. A three-lane plate further allows the cocultivation with more cell types. The passive fluid flow using hydrostatic pressure and the industry-standard layout render the plates fully compatible with robotic pipetting and high-throughput screening. Three-dimensional networks of spontaneously active neurons and supporting glial cells were cultivated recently for up to 6 weeks in the device [46]. The physiologically more relevant culture conditions and the possibilities for high-throughput screening make the system ideal for early drug development and toxicity testing. However, the organ models incorporated are always based within ECM gels. This is of special importance regarding the fact that many pathologies are associated with changes in ECM production that have a strong impact on cell function. Hence, supplying cells with artificial, nonphysiological ECM compositions and structure might lead to cellular aberrations. The standardized plate format impedes organ-specific design constraints, such as the generation of air-liquid interface cultures or cocultures of more than two tissues.

The importance of interconnected multitissue devices and continuous organ-organ crosstalk was shown by Olivier Frey's group from the ETH Zurich and the company InSphero AG (Schlieren, Switzerland) (Figure 2D). Based on their expertise of microtissue formation and cultivation by gravity-enforced cellular self-assembly, a PDMS-based microfluidic system was developed connecting up to eight microtissue spheroids [47,48]. The 96-well plate-based system comprises several separate straight perfusion channels with open medium reservoirs at both ends. Both tissue loading and perfusion are gravity-driven by tilting the device. The use of spheroidal microtissues is advantageous in many ways. They are scaffold-free cell aggregates allowing a tissue-like arrangement of cells during aggregation and, hence, generally show an organotypic phenotype $[49,50]$. Using mixtures of different cell types enables heterotypic cell-cell contacts, further enhancing the tissue-like functionality and differentiated state of cells. Additionally, spheroids can be reliably produced in well-controlled sizes at moderate throughput and the spherical shape makes them easy to handle. Therefore, systems using spheroid models are robust and easy to apply. The proof of concept was performed cultivating rat liver and colorectal tumor microtissues for 8 days in the presence of cyclophosphamide. It demonstrated the importance of interconnected multitissue devices: the prodrug cyclophosphamide reduces tumor growth significantly, but only after bioactivation by the liver. This effect could only be observed in the microfluidic device. The discontinuous transfer of supernatant via pipetting from static liver microtissues treated with cyclophosphamide to tumor tissues did not affect the tumor growth significantly [48]. This again highlights the importance of continuous medium circulation and tissue interaction.

Another development from the ETH combines aggregate formation and chip technology in a single device [51]. It utilizes the hanging drop technology not only for organoid formation, but interconnected droplets also act as a network of interacting cultivation compartments. The platform allows robust and reconfigurable perfusion and effortless multi-organ dosage experiments.

Even though, the combination of various tissue models in a shared media circuit could be achieved by many research groups, in vivo-like tissue-tissue communications still remain an issue for most of them. The artificial media composition and lack of, for example, the nervous or lymphatic systems bear both challenges and chances. From a technical point of view, especially modeling polarized parenchyma such as the liver or organ feedback-loops like the enterohepatic circulation are unsolved endeavors.

\section{Targeting ADME profiles in MPS}

However, not only multitissue interactions, but also physiologically relevant organ volumes and flow rates were shown to be of high value. Shuler et al. presented systems showing in vivo-like tissue-tissue mass ratios, flow rates and fluid retention times in the respective organ compartments. The devices developed in his group are based on physiologically based pharmaco- 
kinetic (PBPK)/pharmacodynamic models. Adhering to PBPK model parameters in device production is of particular importance for studying the ADME profiles of substances for the drug development process. The combination of different tissue cultures in a microfluidic system based on a PBPK model enables the emulation of time-dependent concentrations of a parental compound and its metabolites. Viravaidya and Shuler from the Cornell University in Ithaca (NY, USA) developed a four-compartment chip for cocultures of liver, lung and fat tissue models in 2004 [52]. Since then, many different systems and applications to toxicity testing have been published. Physiologically relevant data for substances such as naphthalene, doxorubicin, tegafur, atorvastatin and valproic acid, just to name a few, were obtained [53-55]. This success inspired many MPS engineers. The number of MPS published based on PBPK models is currently increasing. The most recent system of Miller and Shuler, furthermore, allows the integration of barrier organs, which had not been possible in previous versions [56] (Figure 2E). The spin-off, Hesperos (FL, USA), currently produces only a limited number of standard two-organ systems, but they offer to develop custom designs for almost any number of cell types.

Summarizing, the combination of relevant and highly sophisticated single-organ systems in a common perfusion system designed on physiologically based parameters represents a major gain in the pursuit of relevant test systems. Additionally, endothelial cells are known to establish an instructive vascular niche and induce organogenesis under both in vivo and in vitro conditions [57]. A continuous endothelial network that penetrates even through the tissueengineered organoids will enhance the supply with oxygen and nutrients, as well as the depletion of metabolic products. Endothelial cells, furthermore, act as a barrier controlling the diffusion of small and large hydrophilic molecules and protecting subjacent parenchymal cells from nonphysiological mechanical strain. Therefore, integrating endothelial lining to the microfluidic circuit represents a further step toward in vivo-like cellular crosstalk and morphogenesis. Uwe Marx and his team from the Technische Universität Berlin and TissUse GmbH (Berlin, Germany) presented a microphysiological two-organ system where the closed microchannel circuit was entirely covered on all fluid contact surfaces with human dermal microvascular endothelial cells [58]. Here, a peristaltic on-chip micropump generated pulsatile shear stress in a widely adjustable range enabling long-term in vivo-like behavior of the endothelial cells. Furthermore, the near physiological fluid-to-tissue ratio and the closed microfluidic layout allowed for cell-medi- ated signaling and enriched medium to act on target cells. Strategies were also developed to establish a vascular network inside the integrated organ compartments [58,59]. Having a closed microfluidic network not only throughout the medium channels, but also vascularizing respective organ models of the device would bring many advantages. Size restrictions due to diffusion limits no longer apply to vascularized tissues. Furthermore, such vasculature is required as a barrier for the envisioned integration of an immune system.

The group further worked on the combination of four organ equivalents in a single MPS realizing the ADME profile (Figure 2F) [23]. An integrated skin biopsy resembled the dermal absorption route, while a human primary intestinal barrier model purchased from the company Matek represented an oral administration route. The introduction of barrier tissues relevant for the respective application routes are needed to model the bioavailability of a substance thoroughly. In-house assembled liver aggregates served as prime metabolic organ equivalents, and a proximal tubule barrier provided an excretory route, separating the general circuit from a primary urine compartment. Four-week viability and homeostasis of glucose concentration, lactate dehydrogenase activity and transcriptional markers could be shown. The platform paves the way to future body- or human-on-a-chip devices that have the potential to fully replace animal models in substance testing.

The endeavor to build a test system relevant for drug testing has advanced substantially during the last few years. No MPS strategy has ultimately gained broad acceptance yet, as the application possibilities are similarly broad. Whether any devices will become superior is questionable, after all. Many challenges and complex questions need to be answered, still. Some of which are addressed in the following sections.

\section{Finding the appropriate cultivation conditions in MPS}

The development of MPS implicates new challenges, especially on the level of high-throughput applicability. What works very well on a laboratory level is not necessarily as efficient on a production scale.

Primary cell material (i.e., from isolations of native tissue), for example, often lacks donor availability and suffers from donor variability. Hence, there is the continuous pursuit of new cell sources that mimic the in vivo phenotype robustly. Apart from primary cells, cell lines, artificially immortalized cells or human pluripotent stem cells are often used. The latter encompass both human embryonic stem cells and induced pluripotent stem cells (iPSCs). 
Cell lines are found repeatedly to be not optimal, not necessarily in terms of physiological comparability, but rather in terms of applicability. One third of all cell cultures are thought to have inter- and intra-species contaminations leading to gross misidentification and misinterpretation [60]. Simultaneously, genetic instability $[60,61]$ and mycoplasma contamination [62] interfere with the reliability of cell lines. Their biggest asset is, however, their proliferative capabilities. For the MPS on an industrial level, cell sources should be expandable (i.e. non-phenotypical) to inoculate a number of devices. On the other hand, cells should not overgrow the MPS thereafter. All cells for multi-organ MPS should also be compatible. In other words, tissue engineers are looking for the Swiss Army knife among cell sources.

There is no shortage of innovation, though. The German companies upcyte and InSCREENeX are both reprogramming primary cells, such as hepatocytes, to enable their in vitro expansion [63-65]. The technology can be reversely switched on and off to prohibit expansion in the intended assay [66]. Another approach is the iPSC technology. Dedifferentiated from primary cells, such as fibroblasts, peripheral blood mononuclear cells or urinary epithelial cells, these cells are envisioned to redifferentiate into any cell type. The iPSCs are expanded for this, and differentiation is artificially induced and potentially stabilized in appropriate MPS cocultures. Furthermore, they offer the possibility of modeling diseased cells and ultimately patients (patient-on-a-chip) [67]. When opting for multi-organ devices comprising parts of the immune system, the compatibility between cells becomes a concern. Yet, an MPS with autologous cells is feasible with iPSCs from a single donor [34].

Furthermore, it should be noted that the choice of media is complicated when drafting multicellular or even multitissue systems. Every cell type is cultivated traditionally in a specialized and highly optimized medium designed mostly for fast cell growth rather than parameters relating to true physiological behavior. Growth factors, the addition of fetal serum or serum substitutes and even basal media compositions vary strongly. The application of undefined supplements, such as animal serum, is problematic in terms of lotto-lot variability, standardization, interference with the drugs tested and the maintenance of some cells' differentiation state (this is true especially for progenitor and stem cells). These substances are, thus, highly controversial not only among MPS engineers. Studies are carried out comparing single-tissue cultures and multitissue experiments performed under the same medium conditions. They are of great importance in understanding the models and the right choice of cul- ture conditions. For cocultures, mixtures of cell-specific specialized media, new purpose-made media formulations or media without additives have been used so far $[23,53,68]$. Eventually, scientists are looking for the ubiquitous medium that fits all cell types. In vivo the cells micro-environments are exposed to interstitial fluids with tissue-specific compositions. Emulating this is, however, only possible with a functional vasculature. Only then, an ubiquitous medium would be the equivalent to 'blood' or 'plasma'.

Similarly, the study of the materials used in the generation of the MPS and their influence on cells and (test) substances is of crucial importance. More complex systems are often composed of more than one material. Polydimethylsiloxane has been used frequently, especially among scientists, due to its low cost, ease of fabrication and biocompatibility. It finds its way increasingly into industrial applications. Nevertheless, PDMS is controversially discussed as it is known to adsorb and absorb small hydrophobic molecules and their metabolites. This impedes predicting the fraction of free compound reaching the cells [69]. Thermoplastic polymers, such as PC, PMMA and COC, do not face these issues and are used routinely in cell culture, and protocols for surface treatment are well-established. Here, other constraints such as material stiffness, gas permeability and the effort for design changes arise.

Finally, it is of prime importance to characterize the flow regime in the devices thoroughly. This is especially true when working with endothelial cells in the channels or with shear sensitive cells such as hepatocytes. The characterization of the actual flow in a microfluidic device is often disregarded, although computational models and calculations of flow behaviors often do not reflect reality. This is due mostly to oversimplifications in generating the model or to not taking all the influencing parameters into consideration. A thorough study of systems using techniques such as particle image velocimetry was shown to lead to well-characterized devices $[23,58]$.

\section{Achievements in industrial adoption}

The research into MPS is a very young field and still at a relatively early stage, but new exciting innovations and technologies are developing at an ever-increasing speed. Although there are many challenges and hurdles to be solved using MPS, nearly all major pharmaceutical and consumer products companies are involved in systematic feasibility studies. Some companies have even started to build up small investigational units for organ-on-chip devices in house. Others are in strong collaborations with the companies mentioned above providing commercially available systems. Emulate Inc., for example, announced a strategic research col- 
laboration with Johnson \& Johnson Innovation (NJ, USA) [70]. Using Emulates organ-on-chip platform, the human response of drug candidates is to be predicted and the drug development process improved. Similarly, $\mathrm{CN}$ Bio Innovations announced a research collaboration with an undisclosed pharmaceutical company to perform studies on their full viral lifecycle Hepatitis B model [71]. Cosmetics companies such as Beiersdorf AG (Hamburg, Germany) are already working with MPS models from TissUse GmbH [72]. Companies adopting these systems and using them for in-house decision-making during the drug development process are already currently producing high-value data relevant for later qualification studies. The widespread use of the various MPS platforms will lead to a greater familiarity with and understanding of the benefits and limitations of the technology. Showing that the highquality data derived from these systems are consistent with those derived from other approaches (especially human data) and, furthermore, using them to answer questions that cannot be addressed in animal models will provide further testimony for their reliability.

European flagship projects such as the EU-ToxRisk further support the development of new approaches for mechanism-based toxicity testing and risk assessment. The final goal is a paradigm shift in toxicology toward an animal-free chemical safety assessment. An international consortium of 39 partners has been funded by the European Commission since 2016 to integrate new concepts such as MPS-based systems for regulatory chemical safety assessment. This evaluation of MPS under European programs and the submission of reliable data will aid not only industrial adoption, but also regulatory acceptance. An expert report recently depicted that the industrial adoption period started in 2015 will finally lead the way for regulatory acceptance in 2020 [34].

\section{Regulatory acceptance through validation}

It has always been a challenge for regulators across the world to meet the legislative requirements. Further demands in intensified safety assessments will complicate substance authorization. In vitro methods are intended to handle future risk evaluation. Alternative methods need to fulfill the 'fitness-for-purpose' criteria for regulatory acceptance [12]. Consequently, the EMA gave out three criteria [73]: first, a method needs to be valid, meaning it has a standardized protocol and clear readouts. Further, it needs to be reliable, relevant and validated by recognized institutions. Second, the new method should generate new and meaningful data or replace an existing method with the same or better results. Third, the new method must have been applied in parallel, but independently to an accepted method in a regulatory decision-making process.
To achieve these criteria, first, the qualification of the equipment operating the device according to standard installation, operation and performance qualification is required. Second, the generation of reliable, reproducible and robust data needs to be assured by the adherence to the rules of Good Cell Culture Practice [74], and the use of qualified cell and tissue sources. The improvement of existing models and assays toward a closer recapitulation of in vivo physiology was also recommended recently [75]. Defining the critical regulatory gaps which can be addressed by the MPS is essential to define the biological and functional features of the model system. In addition, the library of reference compounds for qualification should be linked directly to the regulatory question being addressed. Similarly, the 'scientific basis' needs to be properly described. This includes the biological and physiological processes modeled by the test system and thought to be relevant for the substances' adverse effect.

The final validation roadmap might then comprise two different ways depending on the segment and aim of the model. Systems aiming for safety and efficacy testing during drug development might be qualified through the US FDA drug development tool (DDT) Qualification program. This is especially true for systems requiring fast track validation, such as those MPS models targeting compounds or diseases that cannot be tested using techniques currently available. This applies especially to novel biopharmaceuticals specially designed for humans and irrelevant in test animals. Here, a clear description of the manner and purpose of use of the systems as well as its limitations is mandatory. Currently, the FDA is implementing DDT qualification programs for biomarkers and animal models. However, this concept can also be applied to other tools proposed for use in regulatory decision-making, including MPS. Furthermore, the validation of an individual biomarker under the Biomarker Qualification Program on a specific MPS may be a key step toward qualification and acceptance as a tool for therapeutics development. Finally, qualifying an MPS for a specific context of use as a DDT will eventually lead to a tool which may be considered product-independent and may be used for efficacy testing in development programs for multiple investigational drugs for the same disease or condition targeted.

The MPS that aim to replace animal or in vitro models in cosmetics and chemicals safety assessment need to oblige the respective OECD guidelines. The process of identifying the hazard potential is highly standardized. It is recommended that MPS adhere to the OECD guidance document on the validation and international acceptance of new or updated test methods for hazard assessment [76]. Here, high-quality data from MPS that are consistent with results derived from 
other approaches will provide initial evidence for their reliability. However, care must be taken when comparing results gained from human-based MPS with animal studies as the latter might lack sensitivity or specificity. If we, today, validate models based on existing models that are not $100 \%$ reliable, we might pass old flaws to new methods. Cross-validation trials and the assessment of biological baselines of MPS-based approaches would be constructive. Obviously, the comparison to the human situation would be the most precise. However, "human data relating to chemical effects (...) are normally not readily available or need at least to be derived from highly uncertain information (e.g., epidemiological data), involving moreover expert judgement" [12]. The demonstration that the new devices can be used to identify compounds that have failed in clinical trials will generate more confidence in modern MPS technologies.

\section{Living up to high expectations}

The expectations of MPS in risk assessment are high, and the demand is even higher. With the European Cosmetics Directive [20], the European REACH regulation [21] and the new amendment to the US Toxic Substances Control Act [77], animal alternatives are being promoted even by legislators. Simultaneously, vast testing especially of existing chemicals is demanded [8]. However, expectations and reality come to a hard clash when toxicologists require from new technologies what current standards cannot fulfill. A study analyzing noncarcinogenic endpoints in mouse and rat experiments came to the conclusion that the results gained from one species cannot predict with high certainty the outcome in the other species and vice versa [78]. Depending on the organ regarded, the results could be even completely contradictory. The Center of Alternatives to Animal Testing and partners looked into in vivo skin sensitization and eye irritation data available through the REACH database $[79,80]$. They found that the intrareproducibility of the tests never exceeded $95 \%$. The reproducibility even lay at only $73 \%$ for positive-evaluated eye irritants. The inter-reproducibility can be as low as $77 \%$ and as high as $92 \%$. Discrepancies between the tests are also admitted in the respective OECD guidelines [81]. As pointed out by Browne et al. [82]: "Recognizing this variability [of in vivo experiments] is important because it sets realistic expectations as to the performance of any alternative method. (...) It is unrealistic that an alternative method should predict both the true positives as well as account for the associated in vivo experimental variability."

\section{Toward a human-on-a-chip}

The MPS that have been described above and that are already on the market have great potential in the early elimination of toxic drug candidates before moving to animal testing. However, these models only answer specialized questions concerning drug distribution, metabolism and effects on predefined organ systems. A systemic model at organismal-level is required aiming for a fully new substance testing paradigm which eliminates the need for animal testing.

The goal here is not only to cocultivate several physiologically relevant organ models in a combined media circuit, but also to obtain a self-contained organismal homeostasis. To achieve this, it was postulated previously that at least the following ten systems should be present and show in vivo-like interaction: circulatory, endocrine, gastrointestinal, immune, integumentary, musculoskeletal, nervous, reproductive, respiratory and urinary (Figure 3) [34].

Of course, several obstacles, especially at the biological-technical interface, need to be resolved. Exemplarily, so far none of the numerous liver-on-a-chip devices has shown an effective segregation of bile into a secondary compartment outside the blood circuit. Similarly, the fine structure of spleen or kidney could not be artificially recreated. Three-dimensional bioprinting might provide a solution to many of these problems. It allows the reproductive and ordered deposition of diverse cell types and materials, enabling the patterning of complex organoid fine structures and gradients. Nevertheless, spatial resolution, matrix interactions and cell densities need to improve [83].

\section{Enhancing reliability by improving readouts}

The integration of various sensors and actuators enhances the degree of control over these cultures and enable physiologically relevant electro-mechano-biochemical signaling. The readout of the electrical activity of neuronal and cardiac cells, for example, and the electrical stimulation of muscle tissue can be performed with the help of microelectrodes integrated into MPS [84,85]. Similarly, transepithelial electrical resistance (TEER) measurements were used to study the integrity of barrier models in MPS [86]. Sensors based on fluorescent indicators as well as label-free microsensors for $\mathrm{pH}$, dissolved gases and temperature, furthermore, allow for online monitoring of the culture conditions. The integration of mechanical coupling is particularly important for the maintenance of differentiated cells derived from organs that perceive constant physical stimuli. That includes not only shear stress on endothelial cells generated by flowing media, but also directed expansion and compression forces relevant for lung, bone and cartilage [24,58]. Similarly, skin and the GI tract are prone to relevant mechanical loads. Sophisticated MPS have been developed to realize these in vitro [87,88]. However, combining these approaches into one microfluidic device remains challenging. 


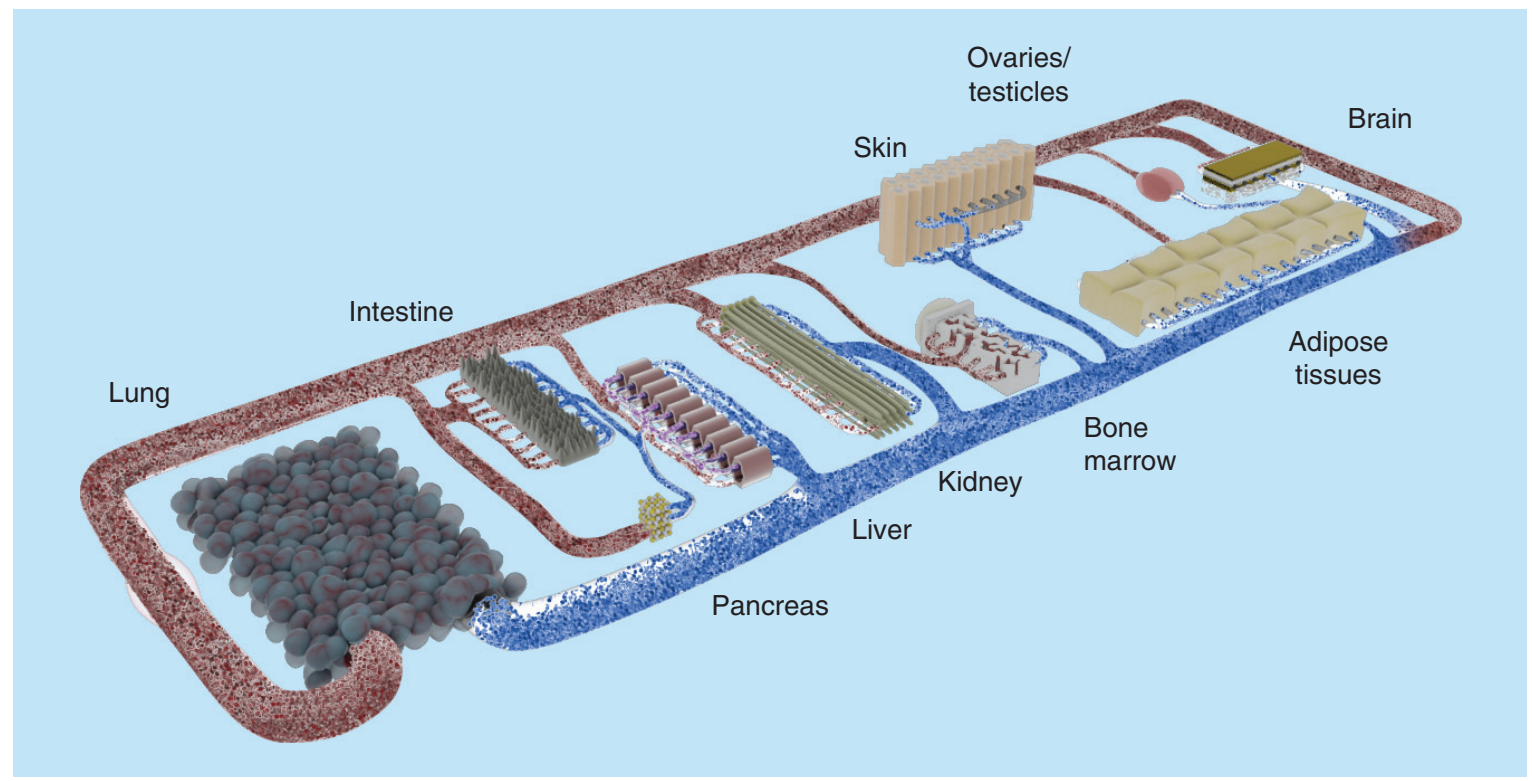

Figure 3. Concept of a human-on-a-chip. The pursuit of the most important bodily functions will lead to a miniature organism on a chip. Common tests conducted in rodents should be realizable with this device. Therefore, the products of the organoids (e.g., urine) need to be discharged in separate compartments. Oral, dermal and intravenous uptake routes, and through inhalation, need to be possible. The transparent device enables optical analysis. Incorporated electrodes will assess barrier resistance, electrophysiological data and key parameters in the supernatants.

The complexity of MPS and human-on-a-chip devices requires the retrieval of as much information as possible from each single experiment. Therefore, highcontent end point analyses, such as RNA sequencing, supernatant metabolite analysis and biomarker measurements, can be performed to characterize the differentiated status of cells and tissues and provide quality control parameters. Combining such readouts with noninvasive sensor-based low content online measurements and microscopic readings can result in a deep insight into tissue biology during multi-organ or human-on-a-chip culture. The complexity is also a reason why interlab reproducibility and transferability has to be tested thoroughly. A high degree of automation is envisioned in the near future to make the systems robust and assays scalable toward industrial needs.

\section{Conclusion \& future perspective}

The MPS have evolved recently combining 3D in vitro cell cultures in microfluidic devices with modern mechanical or electrical actuators and sensors. These systems have already shown major improvements which are physiologically relevant. Furthermore, the singleand multi-organ devices presented allow for a better preselection of effective drugs and more accurate prediction of drug-induced organ toxicity. As long-term organ cultures were shown to be possible, repeated dose toxicity assays are feasible. To date, models for the most prominent delivery routes - oral, dermal and inhalation - are under evaluation in MPS systems and devices allowing for ADME profiles. The experience acquired from these MPS and future developments will eventually lead toward the generation of a miniature organism on a chip.

The intensified adoption of MPS in the pharmaceutical industry should lead within the next 5 years to a faster and more precise selection of targets and compounds during drug discovery and lead optimization. In a next step, the industrial adoption and data generation using MPS should trigger a consecutive regulatory acceptance.

Once human-on-a-chip devices allow for predictive systemic testing, it will no longer be required to involve animals in preclinical development and healthy volunteers in Phase I testing. Although it is an ambitious project, the development of human-on-a-chip systems has been recognized as a promising route toward a paradigm shift in the drug development process.

\section{Acknowledgements}

The authors want to thank P Saunders for the outstanding creative assistance and feedback on the manuscript.

\section{Financial \& competing interests disclosure}

The authors are employees of Tiss Use $\mathrm{GmbH}$, which produces and markets the multi-organ-chip platform. The work has been funded by the German Federal Ministry for Education and Research, GO-Bio Grant Number 0315569. The authors have no other relevant affiliations or financial involvement with any organization or entity with a financial interest in or fi- 
nancial conflict with the subject matter or materials discussed

in the manuscript apart from those disclosed.

No writing assistance was utilized in the production of this manuscript.
Open access

This work is licensed under the Creative Commons Attribution 4.0 License. To view a copy of this license, visit http://creativecommons.org/licenses/by/4.0/

\section{Executive summary}

- The fast progress in microphysiological systems (MPS) development has raised expectations that it might revolutionize substance development and risk assessment. These systems are capable of mimicking the physiological interaction of various interconnected organ models emulating organismal functionality and response to substances.

- Microphysiological systems in general are designed to support a physiological environment for in vitro cultures and emulate human biology at the smallest acceptable scale. Their ability to host 3D organoid constructs in a controlled microenvironment with mechanical and electrophysiological stimuli has been shown previously.

- A 3D coculture of various cell types, as well as physical stimulation of organ models in MPS were shown to enhance physiological relevance.

- Microphysiological systems show broad application possibilities. Hence, the systems reported have a similarly broad technological and biological spectrum. For example, the mode of pumping, the format of the device or the complexity of organ models integrated vary.

- Founding MPS on physiologically based pharmacokinetic/pharmacodynamic models allows one to describe the absorption, distribution, metabolism and excretion profile of a compound quantitatively.

- The integration of a fully closed microvascular network, covering all channel surfaces and penetrating organ models would represent a major step forward toward a self-sustained systemic model system and the utilization of a blood surrogate.

- Primary issues that need to be addressed are the choice of cell source and finding the optimal media composition for multi-organ MPS.

- Major pharma and cosmetics companies are already testing MPS in house. Furthermore, European flagship programs target MPS qualification.

- So far, no MPS achieved regulatory validation. Still, various strategies to facilitate regulatory acceptance exist.

- To achieve the final target of a systemic human-on-a-chip model at organismal-level major questions need to be answered. Still, it holds great potential for a fully new substance testing paradigm eliminating or reducing the need for animal trials.

\section{References}

Papers of special note have been highlighted as:

- of interest; $\bullet \bullet$ of considerable interest

1 Paul SM, Mytelka DS, Dunwiddie CT et al. How to improve R\&D productivity: the pharmaceutical industry's grand challenge. Nat. Rev. Drug Discov. 9(3), 203-214 (2010).

2 DiMasi JA, Grabowski HG, Hansen RW. Innovation in the pharmaceutical industry: new estimates of R\&D costs. J. Health Econ. 47, 20-33 (2016).

3 Hay M, Thomas DW, Craighead JL, Economides C, Rosenthal J. Clinical development success rates for investigational drugs. Nat. Biotechnol. 32(1), 40-51 (2014).

4 Waring MJ, Arrowsmith J, Leach AR et al. An analysis of the attrition of drug candidates from four major pharmaceutical companies. Nat. Rev. Drug Discov. 14(7), 475-486 (2015).

5 Cook D, Brown D, Alexander R et al. Lessons learned from the fate of AstraZeneca's drug pipeline: a five-dimensional framework. Nat. Rev. Drug Discov. 13(6), 419-431 (2014).

6 Kerbrat A, Ferré J-C, Fillatre P et al. Acute neurologic disorder from an inhibitor of fatty acid amide hydrolase. N. Engl. J. Med. 375(18), 1717-1725 (2016).

7 ANSM: Agence Nationale de Sécurité du Médicament. Report by the Temporary Specialist Scientific Committee
(TSSC), "FAAH (Fatty Acid Amide Hydrolase)", on the causes of the accident during a Phase 1 clinical trial in Rennes in January 2016 (2016).

http://ansm.sante.fr/var/ansm_site/storage/original/

8 Hartung T, Rovida C. Chemical regulators have overreached. Nature. 460(7259), 1080-1081 (2009).

9 Long G, Works J. Innovation in the biopharmaceutical pipeline: a multidimensional view (2013). www.analysisgroup.com/uploadedfiles/content/

10 Horner MJ, Hinrichs MJ, Buss N. Safety assessment strategies and predictive safety of biopharmaceuticals and antibody drug conjugates. In: Drug discovery toxicology: From target assessment to translational biomarkers. Will Y, McDuffie JE, Olaharski AJ, Jeffy BD (Eds.). John Wiley \& Sons Inc, 27-38 (2016).

11 Coleman MD, O’Neil JD, Woehrling EK et al. A preliminary investigation into the impact of a pesticide combination on human neuronal and glial cell lines in vitro. PLoS ONE 7(8), e42768 (2012).

12 Griesinger C, Desprez B, Coecke S, Casey W, Zuang V. Validation of alternative in vitro methods to animal testing: concepts, challenges, processes and tools. In: Advances In Experimental Medicine And Biology. Springer 
International Publishing, Eskes C, Whelan M (Eds.), 65-132 (2016).

13 Hackam DG, Redelmeier DA. Translation of research evidence from animals to humans. JAMA 296(14), 1727-1732 (2006)

14 Seok J, Warren HS, Cuenca AG et al. Genomic responses in mouse models poorly mimic human inflammatory diseases. Proc. Natl Acad. Sci. USA 110 (9), 3507-3512 (2013).

15 van der Worp HB, Howells DW, Sena ES et al. Can animal models of disease reliably inform human studies? PLoS Med. 7(3), e1000245 (2010)

16 Leist M, Hartung T. Inflammatory findings on species extrapolations: humans are definitely no $70-\mathrm{kg}$ mice. Arch. Toxicol. 87(4), 563-567 (2013).

17 Greek R, Menache A. Systematic reviews of animal models: methodology versus epistemology. Int. J. Med. Sci. 10 (3), 206-221 (2013).

18 Hartung T. Per aspirin ad astra. Altern. Lab. Anim. 37(Suppl. 2), 45-47 (2009).

19 Woosley RL. Mechanism of the cardiotoxic actions of terfenadine. JAMA 269(12), 1532 (1993).

20 EU: European Union. Regulation (EC) No 1223/2009 of the European Parliament and of the Council of 30 November 2009 on cosmetic products. (2009).

21 ECHA: European Chemicals Agency. The use of alternatives to testing on animals for the REACH regulation. Helsinki (2014). https://echa.europa.eu/about-us/the-way-we-work/

22 Westein E, van der Meer AD, Kuijpers MJE, Frimat J-P, van den Berg A, Heemskerk JWM. Atherosclerotic geometries exacerbate pathological thrombus formation poststenosis in a von Willebrand factor-dependent manner. Proc. Natl Acad. Sci. USA. 110(4), 1357-1362 (2013).

23 Maschmeyer I, Lorenz AK, Schimek K et al. A four-organchip for interconnected long-term co-culture of human intestine, liver, skin and kidney equivalents. Lab. Chip. 15(12), 2688-2699 (2015).

- A four-organ microphysiological system combining the barrier organs skin and intestine with a liver and a kidney model.

24 Huh D, Matthews BD, Mammoto A, Montoya-Zavala $\mathrm{M}, \mathrm{H}$ sin HY, Ingber DE. Reconstituting organ-level lung functions on a chip. Science. 328(5986), 1662-1668 (2010).

25 Maidhof R, Tandon N, Lee EJ et al. Biomimetic perfusion and electrical stimulation applied in concert improved the assembly of engineered cardiac tissue. J. Tissue Eng. Regen. Med. 6(10), e12-e23 (2012).

26 Harink B, Le Gac S, Truckenmüller R, van Blitterswijk C, Habibovic P. Regeneration-on-a-chip? The perspectives on use of microfluidics in regenerative medicine. Lab. Chip. 13(18), 3512-3528 (2013).

27 Pörtner R, Giese C. An overview on bioreactor design, prototyping and process control for reproducible threedimensional tissue culture. In: Drug Testing In Vitro: Breakthroughs and Trends in Cell Culture Technology. Marx U, Sandig V (Eds). Wiley-VCH. (3), 53-78 (2006).
28 Marx U, Walles H, Hoffmann S et al. "Human-on-a-chip" developments: a translational cutting-edge alternative to systemic safety assessment and efficiency evaluation of substances in laboratory animals and man? Altern. Lab. Anim. 40 (5), 235-257 (2012).

29 Grist SM, Schmok JC, Liu MC, Chrostowski L, Cheung KC. Designing a microfluidic device with integrated ratiometric oxygen sensors for the long-term control and monitoring of chronic and cyclic hypoxia. Sensors (Basel) 15(8), 20030-20052 (2015).

30 Ochs CJ, Kasuya J, Liebsch G. au2D-Visualisierung des zellulären Sauerstoff verbrauchs in Mikrofluidiksystemen. BIOspektrum 20(7), 773-775 (2014).

31 Wagner I, Materne E-M, Marx U et al. A dynamic multiorgan-chip for long-term cultivation and substance testing proven by $3 \mathrm{D}$ human liver and skin tissue co-culture. Lab Chip 13(18), 3538-3547 (2013).

32 Powers MJ, Domansky K, Kaazempur-mofrad MR et al. A microfabricated array bioreactor for perfused 3D liver culture. Biotechnol. Bioeng. 78(3), 257-269 (2002).

33 Sin A, Baxter GT, Shuler ML. Animal on a chip: a microscale cell culture analog device for evaluating toxicological and pharmacological profiles. Microfluid. BioMEMS 4560, 98-101 (2001).

34 Marx U, Andersson TB, Bahinski A, Beilmann M, Beken $S$, Cassee FR. Biology-inspired microphysiological system approaches to solve the prediction dilemma of substance testing. ALTEX33(3), 272-321 (2016).

- This review gives a thorough overview of existing microphysiological systems and explains current needs and trends.

35 Hung PJ, Lee PJ, Sabounchi P, Lin R, Lee LP. Continuous perfusion microfluidic cell culture array for high-throughput cell-based assays. Biotechnol. Bioeng. 89(1), 1-8 (2005).

36 Zhang MY, Lee PJ, Hung PJ, Johnson T, Lee LP, Mofrad MRK. Microfluidic environment for high density hepatocyte culture. Biomed. Microdevices 10(1), 117-121 (2008).

37 Sivaraman A, Leach JK, Townsend S et al. A microscale in vitro physiological model of the liver: predictive screens for drug metabolism and enzyme induction. Curr. Drug Metab. 6(6), 569-591 (2005).

38 Gordon S, Daneshian M, Bouwstra J et al. Non-animal models of epithelial barriers (skin, intestine and lung) in research, industrial applications and regulatory toxicology. ALTEX 32(4), 327-378 (2015).

39 Benam KH, Villenave R, Lucchesi C et al. Small airwayon-a-chip enables analysis of human lung inflammation and drug responses in vitro. Nat. Methods 13(2), 151-157 (2016).

40 Jan K-J, Mehr AP, Hamilton GA et al. Human kidney proximal tubule-on-a-chip for drug transport and nephrotoxicity assessment. Integr. Biol. 5(9), 1119-1129 (2013).

41 Korin N, Kanapathipillai M, Matthews BD et al. Shearactivated nanotherapeutics for drug targeting to obstructed blood vessels. Science 337(6095), 738-742 (2012).

42 Jain A, van der Meer AD, Papa A-L et al. Assessment of whole blood thrombosis in a microfluidic device lined by 
fixed human endothelium. Biomed. Microdevices 18(4), 73 (2016).

43 Torisawa Y, Spina CS, Mammoto T et al. Bone marrow-on-achip replicates hematopoietic niche physiology in vitro. Nat. Methods 11(6), 663-669 (2014).

44 Kim HJ, Huh D, Hamilton G, Ingber DE. Human gut-ona-chip inhabited by microbial flora that experiences intestinal peristalsis-like motions and flow. Lab Chip. 12, 2165-2174 (2012).

45 Trietsch JS, Israëls G, Joore J, Hankemeier T, Vulto P. Microfluidic titer plate for stratified 3D cell culture. Lab Chip 13, 3548-3554 (2013).

46 Wevers NR, Van Vught R, Wilschut KJ et al. Highthroughput compound evaluation on 3D networks of neurons and glia in a microfluidic platform. Sci. Rep. 6, 1-10 (2016).

47 Kim J, Fluri DA, Kelm JM, Hierlemann A, Frey O. 96-well format-based microfluidic platform for parallel interconnection of multiple multicellular spheroids. J. Lab. Autom. 20(3), 274-282 (2015).

Kim J-Y, Fluri DA, Marchan R et al. 3D spherical microtissues and microfluidic technology for multi-tissue experiments and analysis. J. Biotechnol. 205, 24-35 (2015).

49 Kelm JM, Fussenegger M. Microscale tissue engineering using gravity-enforced cell assembly. Trends Biotechnol. 22(4), 195-202 (2004).

50 Messner S, Agarkova I, Moritz W, Kelm JM. Multi-cell type human liver microtissues for hepatotoxicity testing. Arch. Toxicol. 87(1), 209-213 (2013).

51 Frey O, Misun PM, Fluri DA, Hengstler JG, Hierlemann A. Reconfigurable microfluidic hanging drop network for multitissue interaction and analysis. Nat. Commun. 5, 1-11 (2014).

52 Viravaidya K, Shuler ML. Incorporation of 3T3-L1 cells to mimic bioaccumulation in a microscale cell culture analog device for toxicity studies. Biotechnol. Prog. 20(2), 590-597 (2004).

53 Oleaga C, Bernabini C, Smith AST et al. Multi-organ toxicity demonstration in a functional human in vitro system composed of four organs. Sci. Rep. 6, 1-17 (2016).

- Novel 4-organ microphysiological system used for toxicity studies in a serum-free defined medium.

54 Viravaidya K, Sin A, Shuler ML. Development of a microscale cell culture analog to probe naphthalene toxicity. Biotechnol. Prog. 20(1), 316-323 (2004).

55 Tatosian D a, Shuler ML. A novel system for evaluation of drug mixtures for potential efficacy in treating multidrug resistant cancers. Biotechnol. Bioeng. 103(1), 187-198 (2009).

56 Miller PG, Shuler ML. Design and demonstration of a pumpless 14 compartment microphysiological system. Biotechnol. Bioeng. 113(10), 2213-2227 (2016).

- A novel human body-on-a-chip system based upon a physiologically based pharmacokinetic-pharmacodynamic model.

57 Ding B-S, Nolan DJ, Butler JM et al. Inductive angiocrine signals from sinusoidal endothelium are required for liver regeneration. Nature 468(7321), 310-315 (2010).
58 Schimek K, Busek M, Brincker S et al. Integrating biological vasculature into a multi-organ-chip microsystem. Lab Chip. 13(18), 3588-3598 (2013).

59 Hasenberg T, Mühleder S, Dotzler A et al. Emulating human microcapillaries in a multi-organ-chip platform. J. Biotechnol. 216, 1-10 (2015).

60 Hughes P, Marshall D, Reid Y, Parkes H, Gelber C. The costs of using unauthenticated, over-passaged cell lines: how much more data do we need? Biotechniques 43(5), 575, 577-578, 581-582 passim (2007).

61 Kleensang A, Vantangoli MM, Odwin-DaCosta S et al. Genetic variability in a frozen batch of MCF-7 cells invisible in routine authentication affecting cell function. Sci. Rep. 6, 28994 (2016).

62 Olarerin-George AO, Hogenesch JB. Assessing the prevalence of mycoplasma contamination in cell culture via a survey of NCBI's RNA-seq archive. Nucleic Acids Res. 43(5), 2535-2542 (2015).

63 Tolosa L, Gómez-Lechón MJ, López S et al. Human upcyte hepatocytes: characterization of the hepatic phenotype and evaluation for acute and long-term hepatotoxicity routine testing. Toxicol. Sci. 152(1), 214-229 (2016).

64 Schaefer M, Schänzle G, Bischoff D, Süssmuth RD. Upcyte human hepatocytes: a potent in vitro tool for the prediction of hepatic clearance of metabolically stable compounds. Drug Metab. Dispos. 44(3), 435-444 (2016).

65 Lipps C, May T, Hauser H, Wirth D. Eternity and functionality - rational access to physiologically relevant cell lines. Biol. Chem. 394(12), 1637-1648 (2013).

66 Ramachandran SD, Schirmer K, Münst B et al. In vitro generation of functional liver organoid-like structures using adult human cells. PLoS ONE 10(10), e0139345 (2015).

67 Bellin M, Marchetto MC, Gage FH, Mummery CL. Induced pluripotent stem cells: the new patient? Nat. Rev. Mol. Cell Biol. 13(11), 713-726 (2012).

68 Maschmeyer I, Hasenberg T, Jaenicke A et al. Chip-based human liver-intestine and liver-skin co-cultures - A first step toward systemic repeated dose substance testing in vitro. Eur. J. Pharm. Biopharm. 95, 77-87 (2015).

69 van Midwoud PM, Janse A, Merema MT, Groothuis GMM, Verpoorte E. Comparison of biocompatibility and adsorption properties of different plastics for advanced microfluidic cell and tissue culture models. Anal. Chem. 84(9), 3938-3944 (2012).

70 Emulate I. Collaboration with J\&J Innovation - Emulate, Inc. (2015).

https://emulatebio.com/press/strategic-collaboration/

71 CN Bio Innovations. CN Bio Innovations marks World Hepatitis Day by signing research agreement with Imperial College London (2014).

http://cn-bio.com/cn-bio-innovations-marks-world-

72 TissUse GmbH. Multi-organ-chips revolutionize drug testing (2014). www.tissuse.com/en/news/press-releases/

73 EMA: European Medicines Agency. Draft guideline on regulatory acceptance of 3 Rs (Replacement, Reduction, 
Refinement) testing approaches. www.ema.europa.eu/docs/en_GB/document

74 Pamies D, Bal-Price A, Simeonov A et al. Good cell culture practice for stem cells and stem-cell-derived models. ALTEX. doi:10.14573/altex.1607121 (2016) (Epub ahead of print).

75 Horvath P, Aulner N, Bickle M et al. Screening out irrelevant cell-based models of disease. Nat. Rev. Drug Discov. 15(11), 751-769 (2016).

76 OECD: Organisation for Economic Co-operation and Development. Guidance document on the validation and international acceptance of new or updated test methods for hazard assessment. No. 34, 1-96 (2005). www.oecd.org/chemicalsafety/testing

77 US-Congress. The Frank R. Lautenberg chemical safety for the 21 st century act (2016).

www.epa.gov/assessing-and-managing-chemicals-under-tsca

78 Wang B, Gray G. Concordance of noncarcinogenic endpoints in rodent chemical bioassays. Risk Anal. 35(6), $1154-1166$ (2015)

79 Luechtefeld T, Maertens A, Russo DP, Rovida C, Zhu H, Hartung T. Analysis of publically available skin sensitization data from REACH registrations 2008-2014. ALTEX33(2), 135-148 (2016).

80 Luechtefeld T, Maertens A, Russo DP, Rovida C, Zhu H, Hartung T. Analysis of Draize eye irritation testing and its prediction by mining publicly available 2008-2014 REACH data. ALTEX33(2), 123-134 (2016).

81 OECD: Organisation for Economic Co-operation and Development. Test No. 429: Skin Sensitisation (2016). www.oecd-ilibrary.org/content/book/
82 Browne P, Judson RS, Casey WM, Kleinstreuer NC, Thomas RS. Screening chemicals for estrogen receptor bioactivity using a computational model. Environ. Sci. Technol. 49(14), 8804-8814 (2015).

83 Murphy SV, Atala A. 3D bioprinting of tissues and organs. Nat. Biotechnol. 32(8), 773-785 (2014).

84 Ahadian S, Ramón-Azcón J, Ostrovidov S et al. Interdigitated array of Pt electrodes for electrical stimulation and engineering of aligned muscle tissue. Lab Chip. 12(18), 3491-3503 (2012).

85 Johnstone AFM, Gross GW, Weiss DG, Schroeder OHU, Gramowski A, Shafer TJ. Microelectrode arrays: a physiologically based neurotoxicity testing platform for the 21st century. Neurotoxicology 31(4), 331-350 (2010).

86 Ferrell N, Desai RR, Fleischman AJ, Roy S, Humes HD, Fissell WH. A microfluidic bioreactor with integrated transepithelial electrical resistance (TEER) measurement electrodes for evaluation of renal epithelial cells. Biotechnol. Bioeng. 107(4), 707-716 (2010).

87 Kim HJ, Huh D, Hamilton G, Ingber DE, Links DA. Human gut-on-a-chip inhabited by microbial flora that experiences intestinal peristalsis-like motions and flow. Lab Chip 12(12), 2165-2174 (2012).

88 Yu J, Peng S, Luo D, March JC. In vitro 3D human small intestinal villous model for drug permeability determination. Biotechnol. Bioeng. 109(9), 2173-2178 (2012).

89 Domansky K, Inman W, Serdy J, Dash A, Lim MHM, Griffith LG. Perfused multiwell plate for 3D liver tissue engineering. Lab Chip 10(1), 51-58 (2010). 